\title{
DESIGN AND REALISATION OF A 100MHz SYNTHESIS CHAIN FROM AN X-BAND REFERENCE SIGNAL
}

\author{
F. Lardet-Vieudrin ${ }^{1}$, P. Salzenstein ${ }^{1}$, D. Vernier ${ }^{1}$, D. Gillet ${ }^{1}$, \\ M. Chaubet ${ }^{2}$ and V. Giordano ${ }^{1}$ \\ ${ }^{1}$ Laboratoire de Physique et de Métrologie des Oscillateurs - CNRS UPR3203, associé à l'Université de Franche- \\ Comté, 32 avenue de l'Observatoire, F25044 Besançon Cedex, FRANCE, Phone: +33 381853974 - Fax: +33 381853998 - \\ e-mail: patrice.salzenstein@1pmo.edu \\ ${ }^{2}$ Centre National d'Etudes Spatiales, 18 avenue Edouard Belin, F33000 Toulouse, FRANCE
}

\begin{abstract}
LPMO has undertaken the building of a cryogenic sapphire oscillator with the support of th french Space and Metrolgy agencies (CNES and BNM). The aim of this project is to provide a reference oscillator presenting short frequency stability better than $5.10^{-14}$ in order to fulfill reference tests requirements for spacial and metrological applications [1]. The cryogenic oscillator can operate on different frequencies ranging from 8 to $13 \mathrm{GHz}$ depending on the sapphire resonator mode chosen as reference. The exact output signal frequency is not 'a priori' known with a great accuracy due to the large relative uncertainties (of the order of $10^{-4}$ ) affecting the resonator frequencies theoritical determination. Then a special synthesis chain has to be designed in order to transfert the cryogenic oscillator performances to a reference signal whose frequency is fully compatible with most of Time and Frequency instrumentation. In this paper, we present the design, realization and preliminary tests of a synthesis chain generating a $100 \mathrm{MHz}$ signal from an $X$-band reference. The performances of the two realyzed prototypes will enable to transfert better than $5^{-10^{-14}}$ short term frequency stability.
\end{abstract}

Keywords - Synthesis, 100MHz, X-band, DDS, Sampling Phase Detector

\section{INTRODUCTION}

Ultra-stable signals are now available in our laboratory from cryogenic sapphire oscillator in X-band [1]. This oscillator has been developped with the support of the Centre National de la Recherche Spatiale (CNES) and Bureau National de Métrologie (BNM) to provide an efficient tool for metrological measurements. Indeed the qualification tests of the new generation of on-board USO and synthesis systems requires the availability of a reference presenting frequency instabilities well below $5.10-{ }^{14}$ on the short term.

Moreover the same reference are needed for the interrogating oscillator used in newly developped frequency standards based on cold atoms [2]. Our cryogenic oscillator is based on a $50 \mathrm{~mm}$ diameter high sapphire resonator operating on a Whispering Galllery Mode (WGM) near liquid Helium temperature. Several quasi transverse magnetic WGM separated by about $600 \mathrm{MHz}$ can be chosen as frequency reference.

Table I gives the frequency and the turn over temperatures of the most interesting modes of our sapphire resonator. The frequency stability of cryogenic oscillator is better than $2.10^{-14}$ for $\tau<100$ s.
TABLE I

FREQUENCIES, TURN-OVER TEMPERATURE, RANK OF THE USEFULL HARMONIC AND BEAT SIGNAL OF THE MOST INTERSTING MODES OF THE SAPPHIRE RESONATOR

\begin{tabular}{ccccc}
\hline $\begin{array}{c}\text { Mode } \\
\text { designation }\end{array}$ & $\begin{array}{c}v \\
(\mathrm{GHz})\end{array}$ & $\begin{array}{c}\mathrm{T}_{0} \\
(\mathrm{~K})\end{array}$ & $\mathrm{n}$ & $\begin{array}{c}\Delta v \\
(\mathrm{MHz})\end{array}$ \\
\hline $\mathrm{WGH}_{13,0,0}$ & 9.747 & 7.33 & 98 & 53 \\
$\mathrm{WGH}_{14,0,0}$ & 10.353 & 7.66 & 104 & 47 \\
$\mathrm{WGH}_{15,0,0}$ & 10.959 & 6.61 & 110 & 41 \\
$\mathrm{WGH}_{16,0,0}$ & 11.565 & 6.42 & 116 & 35 \\
$\mathrm{WGH}_{17,0,0}$ & 12.170 & 6.60 & 122 & 30 \\
$\mathrm{WGH}_{18,0,0}$ & 12.774 & 6.58 & 128 & 26 \\
\hline
\end{tabular}

The transfert of the cryogenic oscillator frequency stability to a $100 \mathrm{MHz}$ signal will be achieved by phase locking a $100 \mathrm{MHz}$ VCXO on a sapphire signal using the scheme given on figure 1 .

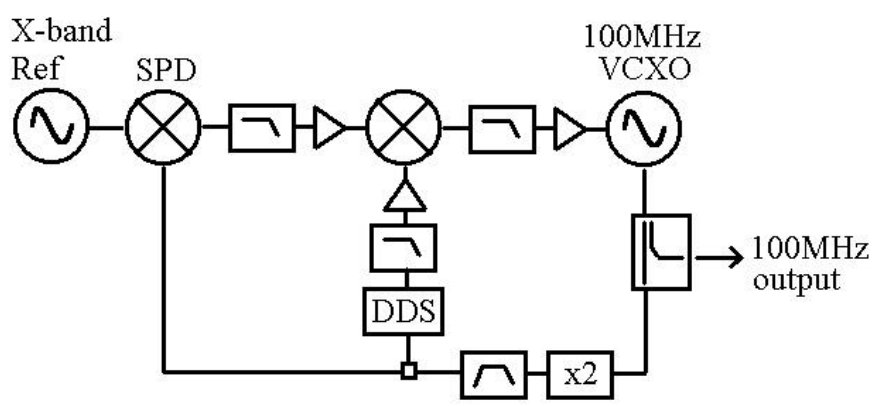

Fig. 1. Principle of the synthesis chain.

Sapphire and a high rank harmonic of the VCXO signals are comparated in a Samplig Phase Detector (SPD) used as a Sampling Mixer, in order to produce a beat signal in the range 20-50MHz. In Table I, the rank $\mathrm{n}$ of the usefull harmonic and the beat signal frequency are given. The PLL error signal is then obtained by mixing the beat note with the output of a Direct Digital Synthesis (DDS) referenced to a $200 \mathrm{MHz}$ signal coming from the VCXO frequency multiplied by 2 , is needed to synthetize signal of to $50 \mathrm{MHz}$. Eventually, this error signal is supplied to the VCXO varicap to lock th loop. The use of a 48 bit DDS enables to achieve frequency of the order of $1.10^{-16}$ compatible with expected frequency accuracy of cold atoms frequency standards. Intrinsic phase noise of the synthesis chain components will limit the short term frequency stability of the $100 \mathrm{MHz}$ outut signal. We present in this paper the measurement of individual components phase noise and the test of the overall system. 


\section{MULTIPLIER}

The $100 \mathrm{MHz}$ signal from the VCXO is multiplied by two by this component. To obtain $200 \mathrm{MHz}$ from the $100 \mathrm{MHz}$ signal, we choose to send this signal through a $90^{\circ}$ hybrid to the LO and RF of a mixer as schematized on figure 2. Actually, this configuration allow the lowest phase noise, compared to commercial multipliers and active multipliers using transistors. This comparizon has been made by the use of interferometric measurements at $100 \mathrm{MHz}$ [3].

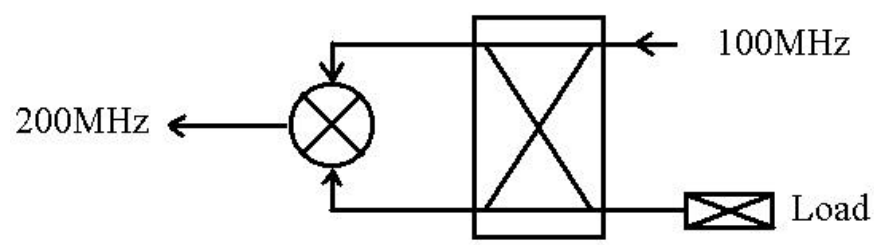

Fig. 2. Principle of the designed multiplier.

The $200 \mathrm{MHz}$ signal is used both to clock the DDS and to provide the LO signal of the SPD. The rejection of $100 \mathrm{MHz}$ pump signal and its harmonics 300 and $400 \mathrm{MHz}$ is better than $65 \mathrm{dBc}$. The phase noise power spectral density of two identical multipliers measured at $200 \mathrm{MHz}$ is given in figure 5 and Table II.

\section{DIRECT DIGITAL SYNTHESIS}

For Direct Digital Synthesis we use an AD9852 commercial model developped by Analog Device Company that uses a 48 bits phase accumulator, a 14 bits ouput converter and a working frequency that can be as higher as $300 \mathrm{MHz}$. This model has been designed for numerical telecommunications applications.

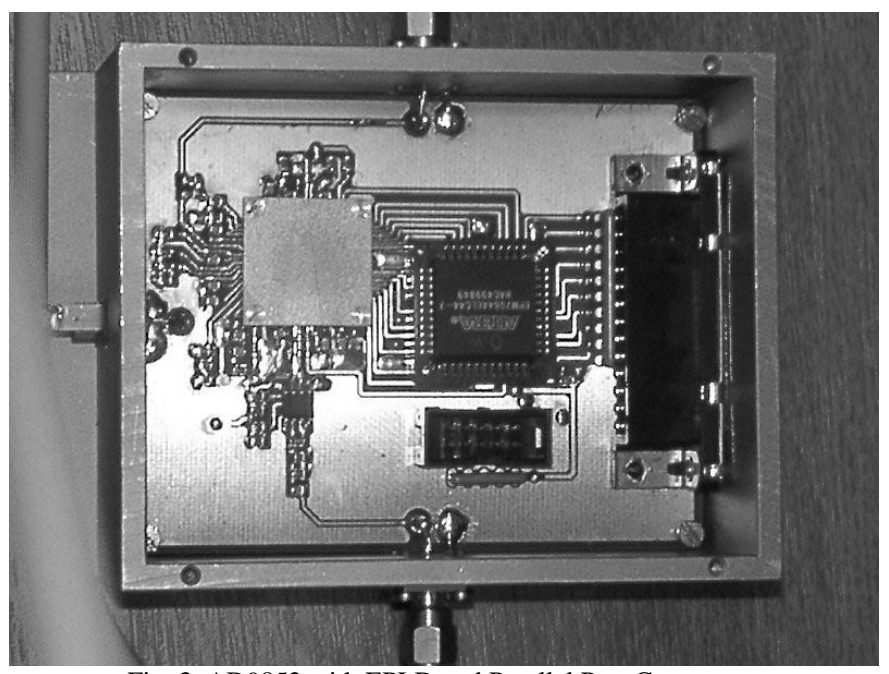

Fig. 3. AD9852 with EPLD and Parallel Port Connector

There are several modes to be used, the fondamental one called single-tone is the most usefull for our application. It generate a sine signal by scrutation of a wave-table and digital-analogic conversion.
The frequency delivered by the DDS is :

$$
\mathrm{F}_{\text {signal }}=\left(\mathrm{p} / 2^{48}\right) \cdot \mathrm{F}_{\text {ref }}
$$

where $\mathrm{p}$ is the incrementation step of the phase accumulator defined as a 48 bits unsigned integer.

As $2^{48}$ is closed to $10^{14.5}$, the frequency resolution at $35 \mathrm{MHz}$ is closed to one microHertz.

We developed a card for the DDS with a driving module based on a Electrical Programable Logic Device (EPLD) that manage the parallel port from a personal computer. It allows the programmation of the DDS registers. In order to drain the heating because of the electrical consumption of the DDS, we put a copper thermal drain bridge that can be seen at the left on the picture. The $35 \mathrm{MHz}$ output signal power is only $-5 \mathrm{dBm}$. Then an amplifier stage has been placed at the DDS output with an anti-aliasing filter. The rejection of the filtering-amplification stage is greater than $40 \mathrm{dBc}$ on the harmonics of the $35 \mathrm{MHz}$ Intermediary Frequency (IF).

\section{SAMPLING MIXER}

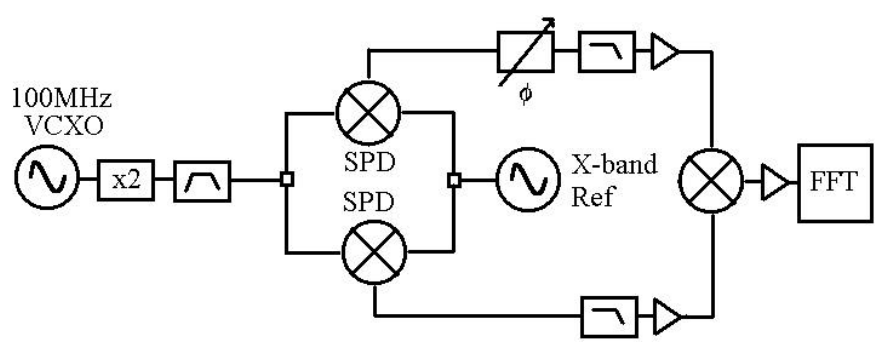

Fig. 4. Principle of the phase noise measurement of the Sampling Mixers.

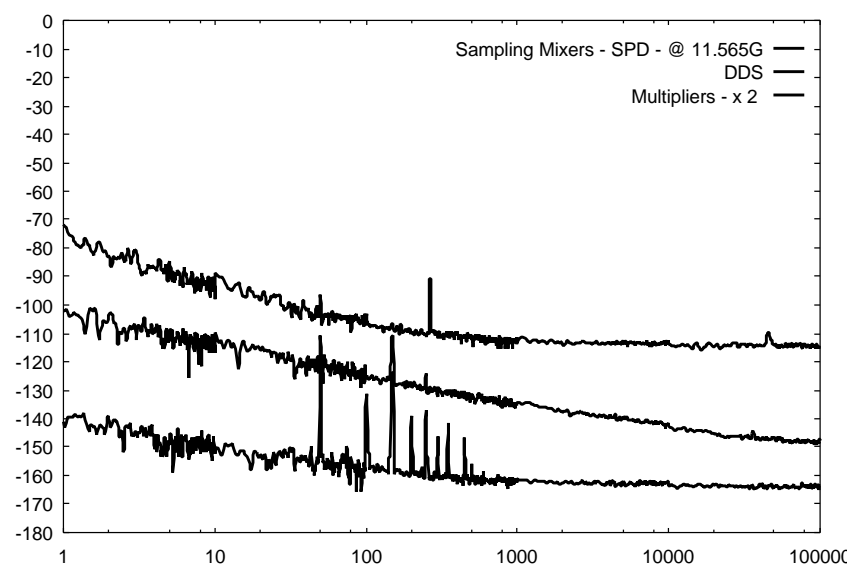

Fig. 5. Spectral density of phase noise $S_{\phi}\left(\mathrm{dB} \cdot \mathrm{rad}^{2} / \mathrm{Hz}\right)$ versus Fourier frequencies $(\mathrm{Hz})$ respectively from the top to the bottom for SPD, DDS and Multipliers.

This component, developped by Avitronics in South Africa, is protected by a radiator in order to operate at room temperature with an internal $36^{\circ} \mathrm{C}$ measured temperature for a $0 \mathrm{dBm}$ microwave input power. Spectral density of phase noise has been measured on two SPD by rejecting the microwave signal delivered by a synthetizer splittered onto both SPDs, and LO pump signal was made of the 
multiplication of the $100 \mathrm{MHz}$ from the VCXO. The $35 \mathrm{MHz}$ output signal phase noise of two SPD is represented in figure 5.

For one SPD, $£(f)=-101 \mathrm{dBc} / \mathrm{Hz}$ at $10 \mathrm{~Hz}$. Between $1 \mathrm{~Hz}$ and $10 \mathrm{~Hz}$, the slope is a little bit higher than a $1 / \mathrm{f}$ slope. It could traduce the sensitivity of the SPD to the $11.5 \mathrm{GHz}$ 'RF' and $200 \mathrm{MHz}$ 'LO' signals. That is why we assume that the SPD introduce a noise $£(f)=-91 \mathrm{dBc} / \mathrm{Hz}$ at $1 \mathrm{~Hz}$.

\section{NOISE PERFORMANCES}

For each components of the synthesis chain, the performances are presented on table II in terms of spectral density of phase noise versus Fourier frequencies.

\section{TABLE II}

SYNTHESIS CHAIN COMPONENT PHASE NOISE VERSUS FOURIER FREQUENCIES

\begin{tabular}{cccccccc}
\hline $\begin{array}{c}\text { Noise } \\
(\mathrm{dBc} / \mathrm{Hz}) \\
\text { versus } \\
\begin{array}{c}\text { Fourier } \\
\text { frequencies }\end{array}\end{array}$ & $\begin{array}{c}\text { Carrier } \\
\text { frequency }\end{array}$ & $1 \mathrm{~Hz}$ & $10 \mathrm{~Hz}$ & $100 \mathrm{~Hz}$ & $1 \mathrm{kHz}$ & $10 \mathrm{kHz}$ & $100 \mathrm{kHz}$ \\
\hline $\begin{array}{c}\text { Oscillator } \\
11,5 \mathrm{GHz}\end{array}$ & $\begin{array}{c}\text { deduced } \\
\text { at } \\
100 \mathrm{MHz}\end{array}$ & -117 & -147 & -177 & $\begin{array}{c}<- \\
177\end{array}$ & $<-177$ & $<-177$ \\
\hline VCXO & $100 \mathrm{MHz}$ & -75 & -106 & -135 & -161 & -176 & -177 \\
\hline $\begin{array}{c}\text { Multiplier } \\
2 \times 100 \mathrm{MHz}\end{array}$ & $200 \mathrm{MHz}$ & -153 & -163 & -172 & -175 & -176 & -176 \\
\hline DDS & $35 \mathrm{MHz}$ & -110 & -120 & -130 & -140 & -149 & $-154,1$ \\
\hline $\begin{array}{c}\text { Sampling } \\
\text { Phase }\end{array}$ & $35 \mathrm{MHz}$ & -91 & -101 & -113 & -120 & -121 & $-121,5$ \\
Detector & & & & & & & \\
\hline
\end{tabular}

\section{NOISE OF THE SYNTHETIZED SIGNAL AT 100MHz}

From the contributions of each element of the synthesis chain, can be deduced the final noise level of the delivered signal at $100 \mathrm{MHz}$. The noise level of the $100 \mathrm{MHz}$ synthetized signal can be estimated from the contributions of each preceding components. Assuming the phase lock loop in operation the error voltage fluctuations $\Delta \mathrm{V}$ is given by:

$$
\Delta \mathrm{V}=\mathrm{K} / \mathrm{p} \cdot\left(\Delta \omega_{\mathrm{i}}-\Delta \omega_{\mathrm{j}}\right)
$$

Where:

$\mathrm{K}$ is the sensitivity of the mixer in $\mathrm{mV} / \mathrm{rad}$ $\mathrm{p}$ is the Laplace's variable, and $\omega_{\mathrm{i}}$ and $\omega_{\mathrm{j}}$ are defined by:

$\Delta \omega_{\mathrm{i}}=\Delta \omega_{\mathrm{Ref}}-\mathrm{n} . \Delta \omega_{0}+\Delta \omega_{\mathrm{x} 2}$

$\Delta \omega_{\mathrm{j}}=\mathrm{m} . \Delta \omega_{\mathrm{S}}-\mathrm{m} / 2 . \Delta \omega_{\mathrm{x} 2}+\Delta \omega_{\mathrm{DDS}}$

$\Delta \omega_{\mathrm{S}}, \Delta \omega_{\mathrm{Ref}}$ are the frequency fluctuations of the locked VCXO and the microwave reference respectively.

$\Delta \omega_{0 \mathrm{DS}}, \Delta \omega_{\mathrm{x} 2}, \Delta \omega_{\mathrm{SPD}}$ are the frequency fluctuations introduced by the DDS, the multiplier by two, the sampling mixer, due to their intrinsic phase noise $\Delta \omega=1 / \mathrm{p} . \Delta \Phi$.

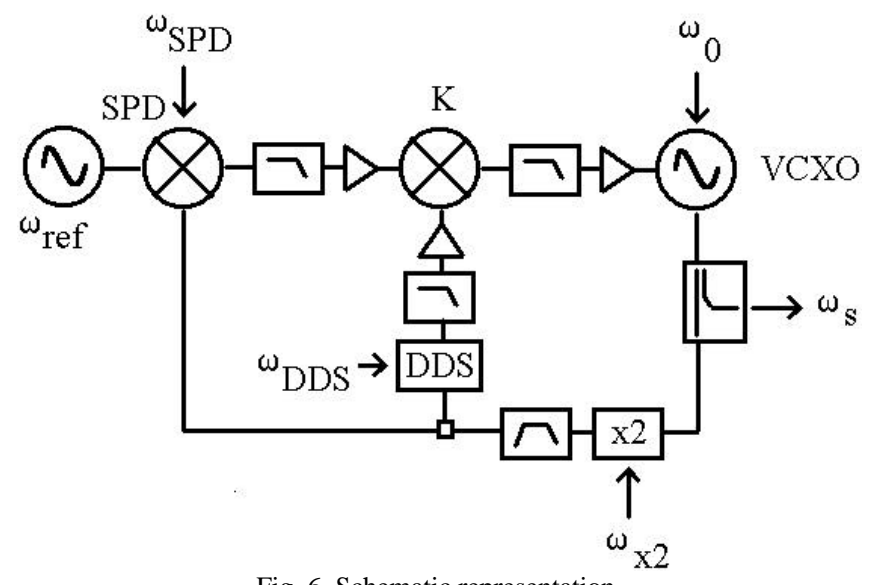

Fig. 6. Schematic representation.

- $\mathrm{n}$ defined in Table I

- $m$ the ratio between the output frequency of the DDS and $100 \mathrm{MHz}$

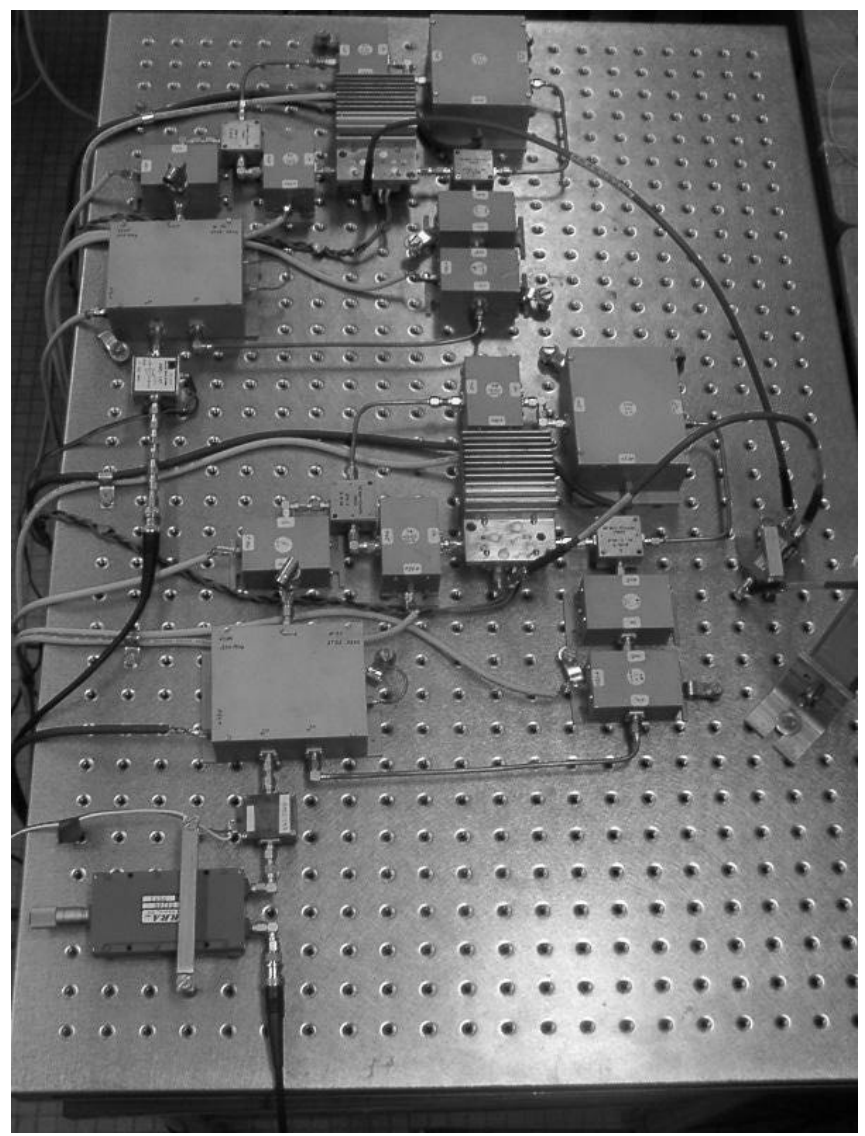

Fig. 7. The two synthesis chains

At the output of the VCXO, the signal is defined by:

$\omega_{\mathrm{S}}=\omega_{0}+\mathrm{K}_{\mathrm{VCO}} \cdot \Delta \mathrm{V}$

Where $\mathrm{K}_{\mathrm{VCO}}$ the VCXO tunning constant in $\mathrm{Hz} / \mathrm{V}$. 
The spectral density of phase noise $S_{\varphi S}$ can then be expressed using (6), by considering a time constant $\tau$ that depend on the characteristic of the synthesis chain:

$S \varphi s=\left(\frac{4 \pi^{2} f^{2} \tau^{2}}{1+4 \pi^{2} f^{2} \tau^{2}}\right) S \varphi_{0}+\left(\frac{1}{1+4 \pi^{2} f^{2} \tau^{2}}\right) \frac{1}{n^{2}}\left(S \varphi_{r e f}+S \varphi_{D D S}+S \varphi_{S P D}+4 . S \varphi_{x 2}\right)$

Noise budget and limit are given in figure 8.

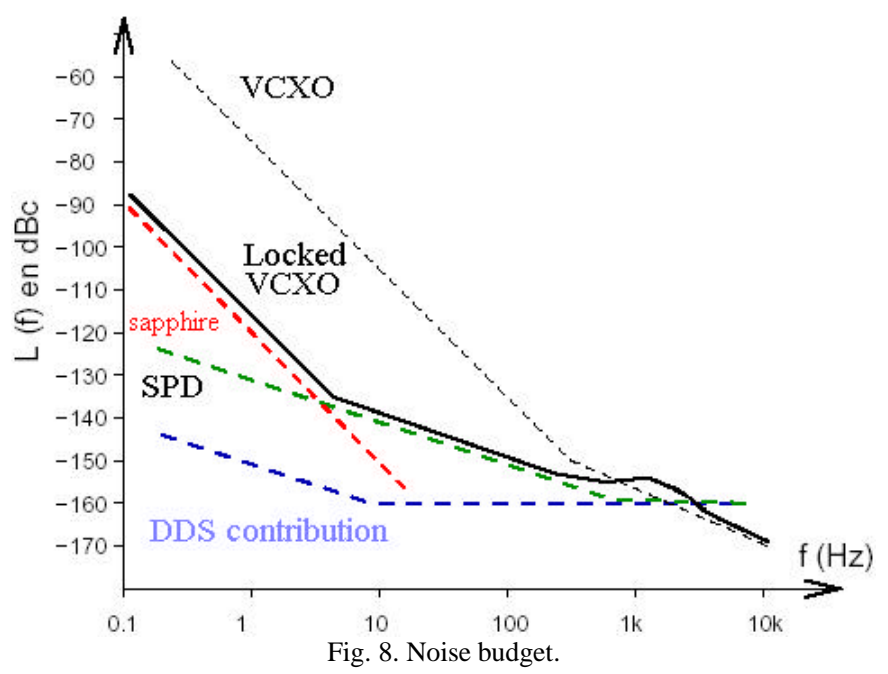

Two identical synthesis chains has been built in order to measure the noise of the system.

Their phase noise was measured by rejecting an X-band CW microwave signal.

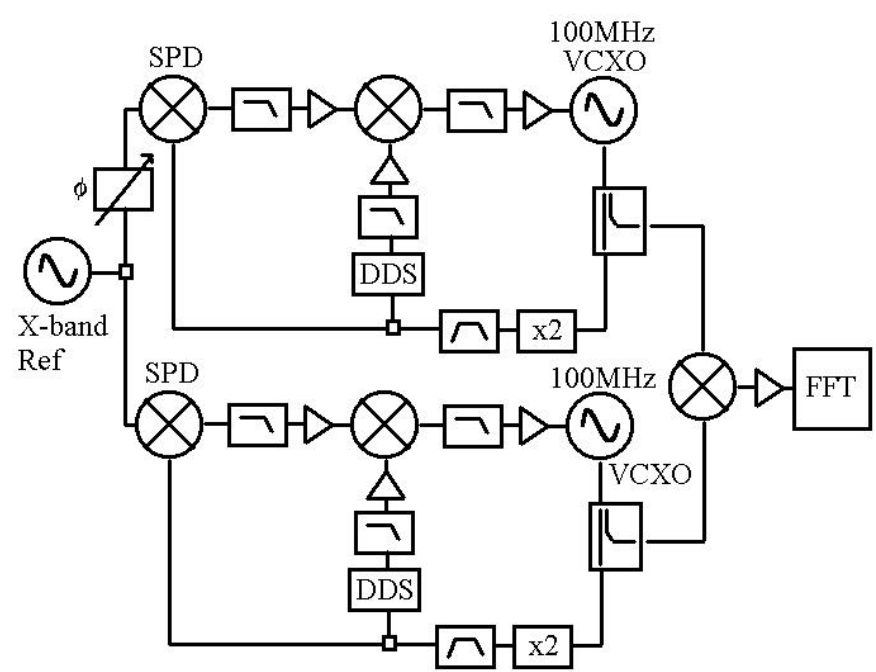

Fig. 9. Principle of the phase noise measurement of the two realized synthesis chains.

Although different frequencies were tested in X-band, we only present here the results for a $11.5 \mathrm{GHz}$ signal for a $0 \mathrm{dBm}$ input power.

The noise of the $100 \mathrm{MHz}$ output frequencies synthetized from the chains is given in the figure 10. For other input frequencies corresponding to the other sapphire resonance mode, the obtained phase noise is about the same.

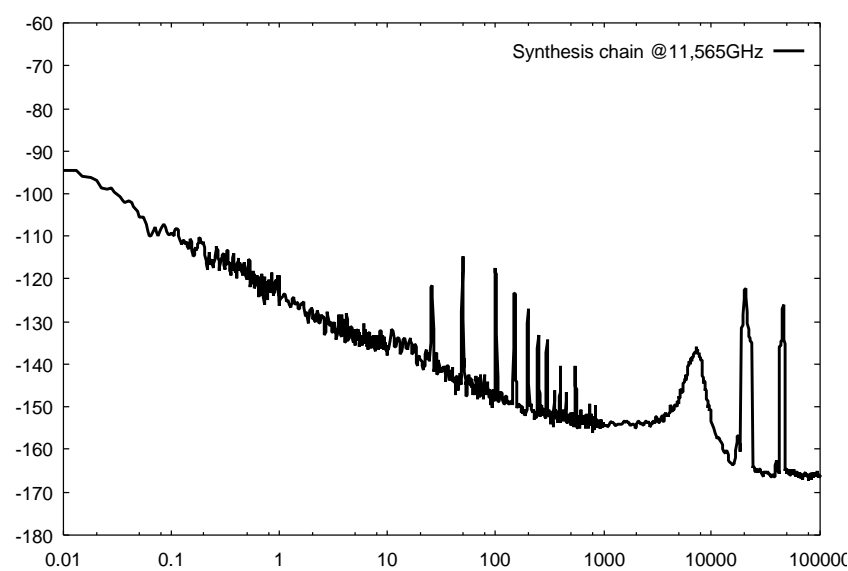

Fig. 10. Performances of the $100 \mathrm{MHz}$ synthetized signals in terms of Spectral density of phase noise $\mathrm{S}_{\phi}\left(\mathrm{dB} \cdot \mathrm{rad}^{2} / \mathrm{Hz}\right)$ versus Fourier frequencies $(\mathrm{Hz})$

\section{DISCUSSION}

By considering the two synthesis chains identical, we deduce the residual phase noise level of the synthetized signal at $100 \mathrm{MHz}$. Results are given versus Fourier frequencies between $0.01 \mathrm{~Hz}$ and $100 \mathrm{kHz}$ on table III.

TABLE III

PHASE NOISE OF THE SYNTHESIS CHAIN

\begin{tabular}{ccccccccc}
\hline Fourier & 0,01 & 0,1 & 1 & 10 & 100 & 1 & 10 & 100 \\
frequencies & $\mathrm{Hz}$ & $\mathrm{Hz}$ & $\mathrm{Hz}$ & $\mathrm{Hz}$ & $\mathrm{Hz}$ & $\mathrm{kHz}$ & $\mathrm{kHz}$ & $\mathrm{kHz}$ \\
\hline $\begin{array}{c}\text { Noise of the } \\
\text { synthetized } \\
\quad \text { signal at }\end{array}$ & -101 & -116 & -131 & -141 & -153 & -160 & -169 & -172 \\
$\quad \begin{array}{l}100 \mathrm{MHz} \\
(\mathrm{dBc} / \mathrm{Hz})\end{array}$ & & & & & & & & \\
\hline
\end{tabular}

For an oscillator in X-band delivering a high stability signal with $\sigma_{y}=2.10^{-14}$ at $\tau=10 \mathrm{~s}$, typicaly obtained in our laboratory for cryogenic resonator-oscillator saphire-based [1], $100 \mathrm{MHz}$ equivalent signal presents a level of noise $£(f)=-120 \mathrm{dBc} / \mathrm{Hz}$ at $1 \mathrm{~Hz}$ from the carrier with a $1 / \mathrm{f}^{3}$ slope. The synthesis chain will be able to reproduce the $100 \mathrm{MHz}$ equivalent signal for Fourier frequencies lower than few Hertz, i.e. frequency stability of the VCXO follows the one of the cryogenic resonator-oscillator for $\tau>1 \mathrm{~s}$. Further from the carrier, the noise performance of the synthesis chain is mainly limited by the noise of the SPD. Moreover, phase lock loop produced a bump in the phase noise level just before Fourier frequency of $10 \mathrm{kHz}$. Further away, the phase noise floor is limited by the VCXO.

\section{REFERENCES}

[1] P.Y. Bourgeois, Y. Kersalé, N. Bazin, M. Chaubet and V. Giordano, Cryogenic opened cavity sapphire resonator for ultra stable oscillator, paper ELL 38056 accepted for publication in Electronics Letters, 2003. 
[2] A. G. Mann, G. Santarelli, S. Chang, A. N; Luiten, Ph. Laurent, C. Salomon, D. G. Blair and A. Clairon, A high stability atomic fountain clock using a cryogenic sapphire interrogation oscillator, Proc. IEEE IFCS, Pasadena, CA, USA, 1998, pp. 13-17.

[3] E. Rubiola and V. Giordano, Advanced interferometric phase and amplitude noise measurements, Review of Scientific Instruments, Vol. 73, No 6, June 2002, pp. 24452457. 